\title{
Percutaneous ultrasound-guided irreversible electroporation: A goat liver study
}

\author{
YING LIU ${ }^{1}$, ZHENGAI XIONG $^{2}$, WEI ZHOU ${ }^{2}$, YUANYUAN HUA $^{2}$, CHENGXIANG LI $^{3}$ and CHENGUO YAO $^{3}$ \\ ${ }^{1}$ Department of Obstetrics and Gynecology, First Affiliated Hospital of Chongqing Medical University, Chongqing 40016; \\ ${ }^{2}$ Department of Obstetrics and Gynecology, Second Affiliated Hospital of Chongqing Medical University, Chongqing 40010; \\ ${ }^{3}$ State Key Laboratory of Power Transmission Equipment and System Security and New Technology, \\ Chongqing University, Chongqing 400044, P.R. China
}

Received April 23, 2012; Accepted June 20, 2012

DOI: $10.3892 / \mathrm{ol} .2012 .781$

\begin{abstract}
Irreversible electroporation (IRE) is a new tumor ablation technique. Pulsed electric fields (PEFs) with permanent duration $(100 \mu \mathrm{sec})$ permanently permeabilize the cell membrane, causing the formation of innumerable permanent nanopores in the cell membrane and leading to cell death. In this study, percutaneous IRE was performed on 24 goat livers under the guidance of ultrasonography (US). IRE-exposed tissues were pathologically examined and glucose-6-phosphatase (G-6-P) and succinodehydrogenase (SDH) staining were used to detect the activity and function of the endoplasmic reticulum and mitochondria of liver tissues at 0 and $24 \mathrm{~h}$ after IRE ablation. Tissue ablation responses were monitored in real-time with US in the percutaneous IRE group; the largest diameter of the ablation zones was measured immediately (D1) and after $24 \mathrm{~h}$ (D2). Following D2 measurement, the animals were sacrificed and the gross sections (D3) were measured. The pathological examination results showed complete tissue necrosis after $24 \mathrm{~h}$ instead of immediately following IRE. The largest long diameters measured by intraprocedural US immediately after IRE (D1, 39.58 $\pm 2.13 \mathrm{~mm})$ were larger than those measured by US after $24 \mathrm{~h}(\mathrm{D} 2,37.07 \pm 3.51 \mathrm{~mm})$ and in gross section measurements (D3, 36.44 $\pm 2.04 \mathrm{~mm} ; \mathrm{P}<0.05)$. D1 showed a good linear correlation with D3 ( $r=0.949)$. We conclude from these studies that IRE is not an acute ablating effect which leads to cell death. If US-guided percutaneous IRE focused on the target liver areas accurately, physicians would be able to assess the extent of necrosis through the regression equation during the IRE ablation procedure, and evaluate whether sufficient electric field energy had been applied to the desired tissue. Assisted with US guiding and monitoring, the
\end{abstract}

Correspondence to: Professor Zhengai Xiong, Department of Obstetrics and Gynecology, Second Affiliated Hospital of Chongqing Medical University, 76 Linjiang Road, Yuzhong District, Chongqing 400010, P.R. China

E-mail: xiongzhengai@126.com

Key words: irreversible electroporation, imaging-guided, ultrasound, tumor, ablation minimally invasive IRE procedure in intraperitoneal lesions may become an important tumor ablation technique.

\section{Introduction}

Electroporation occurs when electric pulses with a field intensity of $\mathrm{kV} / \mathrm{cm}$ and duration of $\mu \mathrm{sec}$ to msec magnitude act on cells, altering the transmembrane potential $(1,2)$. Thus, the lipid bilayer structure is disrupted and small nanopores form in the cell membrane. This allows micro- and macromolecules to be transported into and out of the cells (3-7). Electroporation may be reversible (RE) or irreversible (IRE), according to whether the membrane recovers or not. RE has been used for the delivery of drugs or macromolecules into cells, for example in electrochemotherapy (ECT) $(8,9)$. If the electric field is strong enough, the pores become permanent and cause cell death by interfering with cell homeostasis. As IRE is able to achieve the ablation of desirable tissues without affecting the surrounding normal tissue it has been considered as a novel method for cancer treatment (10).

IRE ablates tumors without chemotherapeutics and is not affected by the heat sink effect. With non-thermal cell death and a markedly decreased treatment time (usually less than 10 min), IRE provides a new ablation technique that may be performed in a well-controlled and focused manner under image monitoring [such as ultrasonography (US)]. In a previous study (11), researchers reported the correlation between US and gross section measurements at the same observation time point (such as $24 \mathrm{~h}$ after IRE ablation). However, IRE is not an acute cell ablation method; tumor cell death occurred sometime after IRE ablation. Therefore, in the IRE ablation, the correlation between the ablation zones of US measurements immediately post-IRE and gross section measurements $24 \mathrm{~h}$ after IRE ablation is important to clinical physicians. The correlation may be used to make a treatment plan prior to the IRE treatment process and to calculate the exact ablation extent using a formula immediately following treatment. In this study, we used an in vivo goat liver model to evaluate the correlation between ablation areas, as measured by US, versus gross section examination and aimed to identify a suitable time point to evaluate the degree of cell necrosis though histopathological changes of goat livers following percutaneous IRE treatment. 


\section{Materials and methods}

Animal care. A total of 24 goats, aged 4-6 months and weighing $25-30 \mathrm{~kg}$, were obtained and maintained by the Division of Laboratory Animal Medicine of Chongqing Medical University (Chongqing, China). All animals received appropriate humane care from properly trained professional staff in compliance with both the Principals of Laboratory Animal Care and the Guide for the Care and Use of Laboratory Animals approved by the Animal Care and Use Committees of Chongqing Medical University.

IRE of goat livers and US measurement. Goats received a general anesthesia: induction was performed using an intramuscular injection of diazepam $(10-20 \mathrm{mg} / 100 \mathrm{~kg})$ and xylazine $(1.5-2.0 \mathrm{ml} / 100 \mathrm{~kg})$. The goats were placed in the supine position following successful anesthesia. The 9-12 ribs which cover the liver tissue had been removed one week prior to surgery. The right upper quadrant and epigastrium were shaved and sterilized in the usual fashion. Pre-ablation US was performed to visualize the normal hepatic anatomy and to locate the desired area for ablation. Using US guidance, we selected sites in all hepatic lobes for ablation.

Electroporation was performed with an electric pulses therapeutic system (manufactured by State Key Laboratory of Power Transmission Equipment and System Security and New Technology, Chongqing University) though electrodes at a frequency of $1 \mathrm{~Hz}$, voltage 2,000 V and pulse duration $100 \mu \mathrm{sec}$. The voltage and wavelength of the electric pulses were monitored throughout the procedures with a TDS3032B oscilloscope (Tektronix, Beaverton, OR, USA).

A total of 24 goats underwent ablation of the normal liver (of those animals, two for sample collection immediately post-IRE ablation, another two goats received lower voltage electric fields at frequency of $1 \mathrm{~Hz}$, pulse duration $100 \mu \mathrm{sec}$, voltage $100 \mathrm{~V}$ as a control group). Each goat was to be ablated at two points, mainly at the right lobe of the liver. Two electrodes with a $2-\mathrm{cm}$ probe distance in the US group were placed into the liver under US guidance through the animal skin to the target areas. The ablating area was oblong in shape and the correlation of long diameters of the ablation zone were measured and analyzed. The long diameter of the ablation zone was monitored and measured in real-time with US at the maximum diameter, along the insert course of the ablation electrode, which was repeated immediately after the procedure (D1) and again $24 \mathrm{~h}$ (D2) after the procedure. A total of 120 pulses were applied through the electrodes.

Tissue collection and gross measurement. The treated area was observed by ultrasound scanning in real-time at 0 and $24 \mathrm{~h}$ after IRE ablation. Goats were heparinized with 5,000 units of heparin and then sacrificed with an overdose of pentobarbital sodium. The livers were harvested and sectioned at a 2-5 $\mathrm{mm}$ thickness along the insert course of the ablation electrode. At gross section examination, the sections of the ablation zones were measured and photographed for comparison with US measurements of the lesions. The largest long diameters of the gross sections (D3) were measured by electric vernier caliper after the animals were sacrificed $24 \mathrm{~h}$ post-IRE ablation.
Table I. Comparison of the measurement under ultrasound and of the gross specimen (mean $\pm \mathrm{SD}$ ).

Maximum ablation zone dimensions

Measurement (mm)

US measurement $0 \mathrm{~h}$ post-

$39.58 \pm 2.13(\mathrm{D} 1)$

imaging-guided IRE ablation

US measurement $24 \mathrm{~h}$ post-

$37.07 \pm 3.51(\mathrm{D} 2)$

imaging-guided IRE ablation

Gross section measurement $24 \mathrm{~h}$

post-imaging-guided IRE ablation

$36.44 \pm 2.04(\mathrm{D} 3)$

IRE, irreversible electroporation.

Histochemical analysis. Each section was divided into two parts for subsequent analysis. One part of the treated area was fixed in $4 \%$ paraformaldehyde and embedded in paraffin for hematoxylin and eosin (HE) stain for histomorphological analysis. The other part of the treated area was stored in liquid nitrogen for glucose-6-phosphatase (G-6-P) and succinodehydrogenase (SDH) staining, to analyze the activity and function of the endoplasmic reticulum and mitochondria, respectively.

Statistical analysis. Statistical analysis was carried out using the software SPSS 11.0 (SPSS, Inc., Chicago, IL, USA). The data are presented as the mean \pm standard deviation (SD). Analysis of variance, bivariate correlation analysis and paired t-test were used for comparing the gross section measurements with US measurements of the IRE ablation zones. $\mathrm{P}<0.05$ was considered to indicate a statistically significant result.

\section{Results}

Histological analysis result. Immediately following IRE ablation, the liver cells became edemic and liver sinusoids in the ablated areas showed congestion (Fig. 1A). The activity and function of the endoplasmic reticulum and mitochondria were not eliminated immediately by the pulsed electric fields (PEFs). Staining for G-6-P and SDH were positive (Fig. 2A and B). Complete hepatic cell death, with a sharp demarcation between the ablated and the non-ablated zones, was well visualized $24 \mathrm{~h}$ after the procedure. The ablation areas were filled with neutrophils and eosinophils (Fig. 1B). Staining for G-6-P and SDH were negative (compared with normal control) $24 \mathrm{~h}$ post-IRE (Fig. 2C and D). In the lower voltage fields group, a few liver cells in the ablation area appeared slightly swollen and distended, but no necrosis was observed (Fig. 1C). Gross section examination and $\mathrm{HE}$ stain revealed the structural integrity of the blood vessels and bile ducts within the IRE-ablated zone (Fig. 3A).

Gross section and US measurement. US imaging-guidance was used to accurately focus on the target area. We were able to visualize and measure the ablation areas of IRE under direct real-time US. Among the three sets of data (D1, D2 and D3), the largest long diameters measured by intraprocedural US immediately following the IRE ablation procedure (D1, 39.58 $\pm 2.13 \mathrm{~mm}$ ) was the largest diameter, 

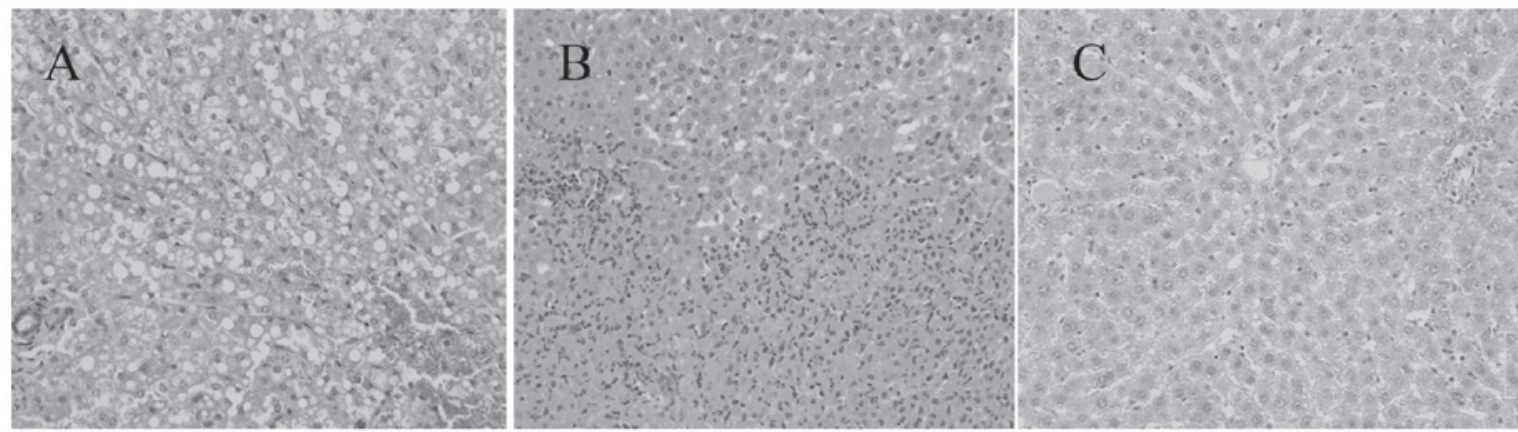

Figure 1. (A) HE staining at $0 \mathrm{~h}$ post-IRE, the liver cells became edemic and sinusoids in the ablated areas showed congestion (x40). (B) HE staining at $24 \mathrm{~h}$ post-IRE. Complete hepatic cell death with a sharp demarcation between the ablated and the non-ablated zones was well visualized and the ablation areas were filled with neutrophils and eosinophils (x40). (C) HE staining for $24 \mathrm{~h}$ post-IRE in the lower voltage field group. A few liver cells in the ablation area appeared slightly swollen and distended, but no necrosis was observed (x40). HE, hematoxylin and eosin; IRE, irreversible electroporation.

A

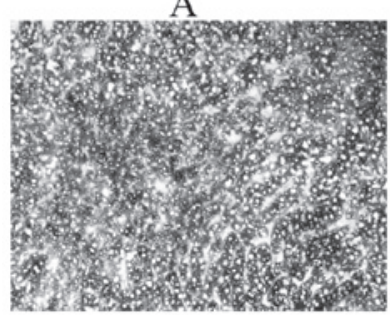

$\mathrm{C}$

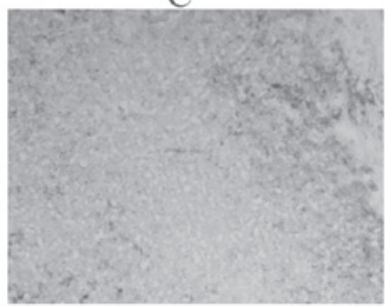

B

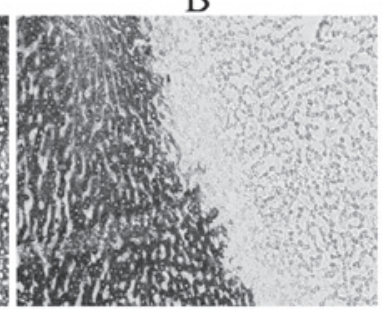

D

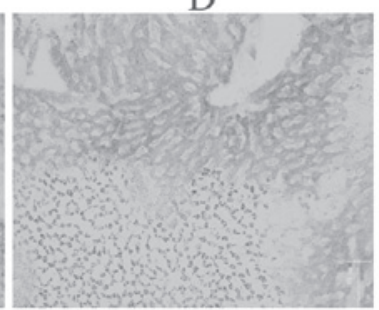

Figure 2. (A and C) The activity and function of the endoplasmic reticulum and mitochondria were not eliminated immediately by PEF. Staining for G-6-P and SDH were positive (x20). (B and D) Staining for G-6-P and SDH were negative (compared with normal control) 24 h post-IRE (x20) There was a sharp demarcation between the ablated and non-ablated zones. PEF, pulsed electric field; IRE, irreversible electroporation; G-6-P, glucose6-phosphatase; SDH, succinodehydrogenase.

followed by the measurement by US $24 \mathrm{~h}$ after the procedure (D2, 37.07 $\pm 3.51 \mathrm{~mm})$. The two sets were significantly different $(\mathrm{P}<0.05)$. The gross section measurement (D3, $36.44 \pm 2.04 \mathrm{~mm}$ ), which was measured $24 \mathrm{~h}$ after imagingguided IRE ablation, was the smallest, but there was no significant difference between D2 and D3 ( $\mathrm{P}>0.05$; Table I). D1 showed a good linear correlation with D3 ( $\mathrm{r}=0.949)$. The regression equation between these two sets of data was $\left[\mathrm{Y}(\mathrm{D} 3)=0.906 \mathrm{X}(\mathrm{D} 1)+0.058, \mathrm{R}^{2}=0.9013\right]$.

The two-dimensional ultrasound imaging revealed that blood vessels were intact and that there was color flow angiography in the blood vessels (Fig. 3B and C).

\section{Discussion}

IRE is a new tumor ablation technique. PEFs with an electric field intensity of at least 500 to $1,000 \mathrm{~V} / \mathrm{cm}$ and permanent duration $(100 \mu \mathrm{sec})$ permanently permeabilize the cell membrane, causing the formation of innumerable permanent

A

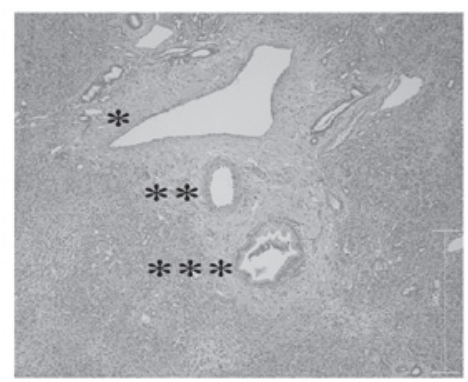

B

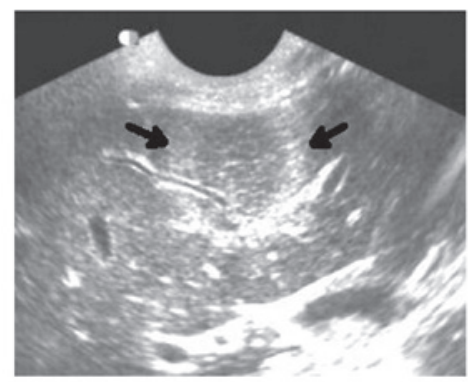

C

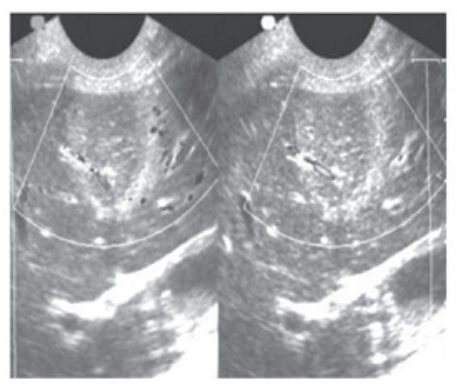

Figure 3. (A) Gross section examination and HE stains revealed the structural integrity of blood vessels and bile ducts within the IRE-ablated zone (x40). "Vein; **artery; ${ }^{* * *}$ bile duct. (B) Two-dimensional ultrasound showed that blood vessels in ablative zone (arrows) were intact. (C) The imaging of Doppler ultrasound showed color flow angiography in the blood vessels. HE, hematoxylin and eosin; IRE, irreversible electroporation.

nanopores in the cell membrane and leading to cell death. IRE has certain unique advantages, such as ablating tissue nonthermally, shortening the ablation time and safety. There is a sharp demarcation between the ablated and non-ablated zones. IRE provides a novel and unique ablation method for cancer treatment.

In this study, we found that IRE ablation of tissues is not an acute effect compared with radiofrequency ablation, 
microwave ablation, cryoablation and high-intensity focused ultrasound. We selected histochemical stain for G-6-P and $\mathrm{SDH}$ to detect the cell viability and $\mathrm{HE}$ stain for histological observation. Enzymohistochemical staining for G-6-P and SDH were used in this study. Researchers usually use G-6-P to detect the activity and function of the endoplasmic reticulum and SDH to detect the activity and function of the mitochondria in liver tissues. When we observed the samples under a microscope immediately post-IRE, it was found that the structure and enzymohistochemical viability of the hepatic cells remained intact. The activity and function of the endoplasmic reticulum and mitochondria were not eliminated immediately by the PEF. Staining for G-6-P and SDH were positive. The complete destruction of hepatic tissue structure and hepatic cell viability was observed through $\mathrm{HE}, \mathrm{G}-6-\mathrm{P}$ and SDH stains in the experiment group $24 \mathrm{~h}$ post-IRE ablation. There was a sharp demarcation between the ablated and the non-ablated zones. However, no necrosis was observed in the control group. Therefore, in the present study, the effective focus area of IRE was observed and measured at $24 \mathrm{~h}$ post-IRE ablation.

In imaging-guided IRE application in the clinic, the accurate focusing on the target area and image monitoring during the process were crucial. Placing the electrodes accurately ensured that the high intensive electric energy ablated the target area effectively and did not injure the surrounding normal tissues. Real-time monitoring by the US may aid the assessment of the extent of the ablation area and observation of tissue response to the electric field energy. Among the three sets of data (D1, D2 and D3), the long diameter measured by intraprocedural US immediately after the IRE ablation procedure (D1) was the largest, followed by the data measured by US $24 \mathrm{~h}$ after the procedure (D2). The two sets of data were significantly different $(\mathrm{P}<0.05)$. The levels of intracellular water molecules increased following the opening of the transmembrane pores by the high voltage of electroporation immediately after IRE ablation. The ablating area became edemic, which was likely caused by increased water content in the area of ablation as a result of the disruption of cellular homeostasis. However, the extent of the ablation area at $24 \mathrm{~h}$ decreased due to hepatic cell necrosis and dehydration. The US measurement of D2, $24 \mathrm{~h}$ post-IRE ablation, is more accurate for calculating the necrotic area.

Another important finding of this study was the accuracy of imaging in guidance and monitoring the extent of the ablation area. We compared the maximum diameters of US-guided IRE ablation areas $24 \mathrm{~h}$ post-IRE ablation measured by US (D2) with its gross section measurement (D3). There was no significant difference between the two sets of data $(\mathrm{P}>0.05)$. The result showed that imaging-guided IRE ablation measured the target area accurately.

In a previous study (11), researchers reported the correlation between US and gross section measurements at the same time point (such as $24 \mathrm{~h}$ after IRE ablation). However, in the development of IRE ablation clinical application, it may be more important to calculate the exact necrotic extent of the ablation areas during the imaging-guided IRE ablation therapy procedure. This determines whether the ablating dosage (voltage and pulses) and electric field intensity are sufficient to completely ablate the lesion of the tumor tissue. Therefore, investigating the correlation between the maximum diameters of the IRE lesions measured by intraprocedural US immediately after the IRE ablation procedure (D1) and gross section measurement $24 \mathrm{~h}$ after IRE ablation (D3) when the animals were scarified was necessary. The results may be used to make a treatment plan prior to the IRE treatment process and to calculate the exact ablation extent using a formula during the IRE process. The statistical results indicated that D1 showed a good linear correlation with D3 $(r=0.949)$. The regression equation between these two sets of data was $[Y(D 3)=0.906 X(D 1)+0.058$, $\left.\mathrm{R}^{2}=0.9013\right]$. Therefore, physicians may be able to assess the exact extent of necrosis using the regression equation during the imaging-guided IRE ablation treatment procedure, and decide whether complementary ablation energy should be added to the lesion areas. Intraprocedural US may not only be used for IRE ablation guidance, but also for measuring and regulating the ablation extent. However, this study had some limitations. The experimental data were not sufficient and the study was a primary investigation of the regression equation between D1 and D3.

With advantages of real-time monitoring, practicability, non-thermal effect and well-controlled ablating range, percutaneous IRE provides a novel and unique method in ablating living cells with lipid bilayer membranes. The activity and function of endoplasmic reticulum and mitochondria in hepatic cells were lost at the same time. Previous studies found that $(12,13)$ the IRE ablation technique is not affected by the heat sink effect of blood flow, which ensured effective transmission and accumulation of electric field energy. In our study, we found that the IRE ablation method destroyed capillaries and cholangioles, but retained vital structures such as the hepatic arteries, hepatic veins, bile ducts and perivascular elastic and collagen fiber structure in the ablation zone $(2,14-16)$. Gross section examination and HE stain showed the structural integrity of blood vessels and bile ducts in the IRE-ablated zone. The two-dimensional ultrasound imaging also showed that blood vessels were intact and that there was color flow angiography in the blood vessels. Thus, IRE produced a more complete and safer tumor ablation effect within the lesion area and did not damage the integrity and basic function of blood vessels in the target area.

\section{Acknowledgements}

This study was supported by the Key Program of National Natural Science Foundation of China (NSFC 50637020).

\section{References}

1. Rubinsky B: Irreversible electroporation in medicine. Technol Cancer Res Treat 6: 255-260, 2007.

2. Davalos RV, Mir IL and Rubinsky B: Tissue ablation with irreversible electroporation. Ann Biomed Eng 33: 223-231, 2005.

3. Weaver JC: Electroporation: A dramatic, nonthermal electric field phenomenon. In: Proceedings of the First World Congress for Electricity and Magnetism in Biology and Medicine. Academic Press, Lake Buena Vista, Florida, 1992.

4. Weaver JC and Chizmadzhev YA: Theory of electroporation: a review. Bioelectrochem Bioenerg 41: 135-160, 1996.

5. Weaver JC: Electroporation of cells and tissues. IEEE Trans Plasma Sci IEEE Plasma Sci Soc 28: 24-33, 2000.

6. Neumann E and Rosenheck K: Permeability changes induced by electric impulses in vesicular membranes. J Membr Biol 10: 279-290, 1972 . 
7. Crowley JM: Electrical breakdown of biomolecular lipid membranes as an electromechanical instability. Biophys J 13: 711-724, 1973.

8. Mir LM: Nucleic acids electrotransfer-based gene therapy (electrogenetherapy): Past, current and future. Mol Biotechnol 43: $167-176,2009$.

9. Neumann E, Schaefer-Ridder M and Wang Y: Gene transfer into mouse lyoma cells by electroporation in high electric fields. EMBO J 1: 841-845, 1982.

10. Sersa G, Miklavcic D, Cemazar M, Rudolf Z, Pucihar G and Snoj M: Electrochemotherapy in treatment of tumours. Eur J Surg Oncol 34: 232-240, 2008.

11. Lee EW, Chen C, Prieto VE, Dry SM, Loh CT and Kee ST: Advanced hepatic ablation technique for creating complete cell death: irreversible electroporation. Radiology 255: 426-433, 2010
12. Daniels C and Rubinsky B: Electrical field and temperature model of nonthermal irreversible electroporation in heterogeneous tissues. J Biomech Eng 131 071006, 2009.

13. Maor E, Ivorra A and Rubinsky B: Non thermal irreversible electroporation: novel technology for vascular smooth muscle cells ablation. PLoS One 4: e4757, 2009.

14. Rubinsky B, Onik G and Mikus P: Irreversible electroporation: a new ablation modality - clinical implications. Technol Cancer Res Treat 6: 37-48, 2007.

15. Maor E, Ivorra A, Leor J and Rubinsky B: The effect of irreversible electroporation on blood vessels. Technol Cancer Res Treat 6: 307-312, 2007.

16. Lee EW, Loh CT and Kee ST: Imaging guided percutaneous irreversible electroporation: ultrasound and immunohistological correlation. Technol Cancer Res Treat 6: 287-294, 2007. 